\title{
Prognostic significance of neutrophil-to-lymphocyte ratio in middle thoracic esophageal squamous cell carcinoma patients undergoing radical esophagectomy
}

\author{
Jia Hu, Dongni Chen, Shaoyong Wu, Youfang Chen, Rongzhen Li, Huikai Miao, Zhesheng Wen \\ Department of Thoracic Oncology, State Key Laboratory of Oncology in South China, Collaborative Innovation Center for Cancer Medicine, Sun \\ Yat-sen University Cancer Center, Guangzhou 510060, China \\ Contributions: (I) Conception and design: J Hu, Z Wen; (II) Administrative support: None; (III) Provision of study materials or patients: None; (IV) \\ Collection and assembly of data: J Hu, D Chen; (V) Data analysis and interpretation: J Hu; (VI) Manuscript writing: All authors; (VII) Final approval \\ of manuscript: All authors. \\ Correspondence to: Zhesheng Wen. Department of Thoracic Oncology, State Key Laboratory of Oncology in South China, Collaborative Innovation \\ Center for Cancer Medicine, Sun Yat-sen University Cancer center, 651 Dongfengdong, Guangzhou 510060, China. Email: wenzhsh@sysucc.org.cn.
}

Background: It is widely accepted that the pretreatment neutrophil-to-lymphocyte ratio (NLR) is an independent predictor of prognosis in multiple malignancies, including esophageal squamous cell carcinoma (ESCC). However, its predictive value in middle thoracic esophageal carcinoma is still unclear. Therefore, the purpose of this study was to investigate the preoperative serum levels of NLR in middle thoracic esophageal carcinoma patients to clarify their clinical significance as predictors of prognosis.

Methods: This study investigated 556 patients with middle thoracic ESCC treated by esophagectomy from January 2010 to December 2012. The prognostic impact of serum NLR level was analyzed. A receiver operating characteristic (ROC) curve was used to identify the NLR for predicting survival. Correlation between the NLR and clinicopathological characteristics was analyzed by $\chi^{2}$ test. Prognostic influence was calculated by using the Kaplan-Meier method and the difference was compared by log-rank test. Cox regression analysis was performed to evaluate the significant prognostic factors.

Results: The cutoff value for the NLR was $2.43 \mathrm{ng} / \mathrm{mL}$, the area under the curve was $0.553(95 \%$ CI: $0.504-0.601 ; \mathrm{P}=0.035)$, and the sensitivity and specificity were $53.3 \%$ and $58.7 \%$ respectively. It is demonstrated that preoperative NLR $(\mathrm{P}=0.003)$, T stage $(\mathrm{P}<0.001)$, N stage $(\mathrm{P}<0.001)$, surgical approach $(\mathrm{P}=0.004)$, and gender $(\mathrm{P}=0.008)$ were independent prognostic factors in middle thoracic ESCC by univariate analysis. Multivariate analysis showed that preoperative NLR $(\mathrm{P}=0.036), \mathrm{T}$ stage $(\mathrm{P}=0.004), \mathrm{N}$ stage $(\mathrm{P}<0.001)$, surgical approach $(\mathrm{P}=0.002)$, and age $(\mathrm{P}=0.019)$ were independent prognostic factors for survival.

Conclusions: Pretreatment NLR $>2.43 \mathrm{ng} / \mathrm{mL}$ could serve as an indicator of poor prognosis in middle thoracic ESCC patients after surgical treatment.

Keywords: Middle thoracic esophageal squamous cell carcinoma; neutrophil-to-lymphocyte ratio (NLR); prognostic factor

Submitted Oct 24, 2019. Accepted for publication Dec 31, 2019.

doi: $10.21037 /$ jtd.2020.01.31

View this article at: http://dx.doi.org/10.21037/jtd.2020.01.31 


\section{Introduction}

Esophageal cancer (EC) is the cancer with the sixth highest incidence and the fourth highest mortality in China (1). The two major pathological types of EC are squamous cell carcinoma (SCC) and adenocarcinoma. Epidemiological studies have demonstrated that the morbidity of adenocarcinoma has increased and now it is the most common pathological type of EC in Western countries, especially among Caucasian. On the other hand, SCC comprises approximately $90 \%$ of all EC cases in China (2). It is demonstrated that esophageal carcinoma is one of the most aggressive malignancies and has a poor prognosis, for the 5-year survival remains low. Thus, reliable prognostic indicators are needed to improve treatment recommendations and prognosis.

The prognostic factors in most gastrointestinal cancers have generally been agreed and include tumor size, lymph node (LN) metastasis, and distant metastasis $(2,3)$. However, the role of routine examination such as computed tomography (CT) or endoscopic ultrasonography in early detection of minimal LN metastasis is limited. Therefore, it is urgently required to identify useful biomarkers to individually predict the therapy effect and prognosis (4). In the past few decades, it has been noticed that the association between inflammation and tumor development has drawn a great deal of attention (5). It is reported that elevated systemic inflammation is correlated with poorer outcome after tumor development (6-8). The ratio of neutrophils to lymphocytes (NLR) refers to the ratio of neutrophils to lymphocytes, which is used to evaluate the potential system balance between neutrophil dependent pre-tumor inflammation and lymphocyte related anti-tumor immune response (9). In previous studies, hematological inflammatory markers such as C-reactive protein, albumin, neutrophils helped to predict survival in patients with different kinds of cancers (10-12). Among these predictors, preoperative NLR is a repeatable and widely used laboratory tumor marker in routine clinical practice. Neutrophils are considered to be the main source of circulating vascular endothelial growth factor, which plays a critical role in tumor-associated angiogenesis (11-13). In addition, neutrophils can promote the production of tumor necrosis factor, interleukin-1, interleukin-6 and other inflammatory cytokines, and provide a good microenvironment for tumor survival and proliferation (14). In contrast, lymphocytes play an important role in tumor specific immune response (15). It has been reported that increased infiltration of lymphocytes in tumor tissue is associated with good prognosis (16). The NLR plays an essential role in cancer diagnosis and prognosis. Accumulating evidence has demonstrated that Inflammation plays an important role in the formation, development and progress of cancer by promoting angiogenesis, promoting life and preventing apoptosis (9).

The higher NLR may represent the trend of increase of pre-tumor inflammation and decrease of antitumor immunity (17). Hence, it is undeniable that the preoperative NLR increase is related to the poor prognosis of different types of cancer patients (14,18-22). However, as no consensus has been reached about the prognostic significance of NLR in middle thoracic ESCC, we designed this retrospective study to investigate the relationship between preoperative serum NLR and prognosis in patients with middle thoracic esophageal squamous cell carcinoma (ESCC).

\section{Methods}

\section{Study population and data collection}

From January 2010 to December 2012, 1281 patients with esophageal cancer (EC) underwent radical resection in the cancer center of Sun Yat-sen University were analyzed retrospectively. The inclusion criteria for this cohort study are as follows: (I) the diagnosis of middle thoracic EC was confirmed by histopathology or cytology; (II) preoperative serum albumin, peripheral lymphocyte count, blood sugar, and hemoglobin were assessed within 1 week. The exclusion criteria were as follows: (I) no clear histopathological or cytological diagnosis of middle thoracic ESCC; (II) presence of other diseases of the blood and immune system; (III) presence of a second primary tumor; (IV) presence of acute or chronic infection; (V) lack of preoperative serum albumin, peripheral lymphocyte count, blood sugar, and hemoglobin within a week; (VI) patients with defective data or who lost follow-up were also excluded. The final study population consisted of 556 patients. All patients received written informed consent.

We evaluated the hematological and biochemical profiles of each middle thoracic ESCC patients. Preoperative serum albumin, peripheral lymphocyte count, blood sugar, and hemoglobin within a week were examined. According to the TNM classification of the eighth edition of the International Union Against Cancer (UICC) and the American Joint Committee on Cancer (AJCC), the 
pathological stage and LN involvement were evaluated (23).

\section{Patient follow-up}

Following surgery, it is suggested that patients should visit the outpatient department every 3 months for the first 2 years, and every 6 months for the next 3 years, and then every year. Patients were observed from diagnosis until death or through November 2018, including a review of clinical attendance records or telephone interviews with patients or their families. The mean follow-up time was 35.0 months (range from 1-75 months).

\section{Statistical analysis}

The endpoint of the study was overall survival (OS), which was defined as the number of days from the date of diagnosis to the date of any cause of death or the date of the last follow-up. Disease free survival (DFS) refers to the time from radical surgery (R0 resection) to the first local recurrence or distant metastasis of ESCC, in order to prevent unrelated causes of death from affecting survival. Logistic regression analysis of the NLR in middle thoracic ESCC showed that a high NLR was an independent risk factor of poor prognosis (OR $=1.132,95 \%$ CI: 1.019 to $1.259, \mathrm{P}=0.021)$. The cutoff value for the NLR was determined by a receiver operating characteristic (ROC) curve and the Youden index. Levels above the cutoff values were defined as high, while those below the cutoff value were defined as low. A $\chi^{2}$ test was used to compare categorical variables. Continuous variables were compared by analysis of variance (ANOVA). Kaplan Meier method was used to analyze the survival curve, and log rank test was used to evaluate the difference of survival rate among groups. In order to identify significant prognostic factors, we conducted multivariate analysis with a Cox proportional hazards model. All calculations were performed using SPSS 21.0 software (SPSS, Chicago, IL) and R (version 3.3.0; http://www.Rproject.org), and a P value of less than 0.05 was considered significant. The method section has been reported in accordance with the STROCSS guidelines (24).

\section{Results}

\section{Demographic data}

We retrospectively analyzed 556 patients with middle

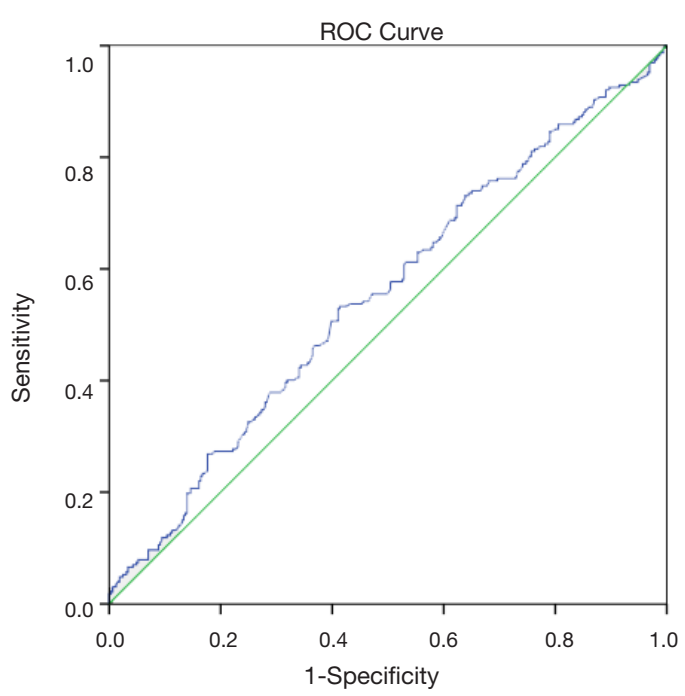

Figure 1 Receiver operating characteristic were employed to assess the serum levels of NLR discriminative performance of the prognosis. NLR, neutrophil-to-lymphocyte ratio.

thoracic ESCC and all patients underwent radical esophagectomy for ESCC. There are 420 male and 136 female patients included in the study, and the median age was 59 years old (range from 28 to 84 years old). According to the maximum Youden index, $2.43 \mathrm{ng} / \mathrm{mL}$ was selected as the best truncation value of NLR, with a curve area of 0.553 (95\% CI: 0.504-0.601; $\mathrm{P}=0.035$; Figure 1), a sensitivity of $53.3 \%$ and a specificity of $58.7 \%$ (Figure 1). 556 patients were divided into two groups: high $(>2.43 \mathrm{ng} / \mathrm{mL} ; \mathrm{n}=256)$ and low ( $\leq 2.43 \mathrm{ng} / \mathrm{mL} ; \mathrm{n}=300)$ NLR.

\section{Baseline characteristics of patients and NLR}

Table 1 shows the relationship between the NLR and baseline characteristics of patients with mid thoracic ESCC. Comparison of clinical and pathological factors between the two groups by analysis of variance (ANOVA). Based on the mean hospitalization 24 days, we divided the patients into two groups. We found significant correlations between the NLR and gender $(\mathrm{P}=0.002)$, $\mathrm{T}$ (tumor) stage $(\mathrm{P}=0.004)$, hospitalization days $(\mathrm{P}=0.049)$ (Table 1). However, there were no significant difference in age distribution, $\mathrm{N}$ stage, TNM stage, surgical approach, complication, blood pressure, pulse rate, respiratory rate, blood loss and postoperative survival rate between the two groups according to the NLR cutoff (all $\mathrm{P}>0.05$ ). 
Table 1 Baseline characteristics of patients stratified for the cutoff point of the levels of NLR

\begin{tabular}{|c|c|c|c|c|}
\hline \multirow{2}{*}{ Variables } & \multicolumn{2}{|c|}{ The level of NLR } & \multirow{2}{*}{ Total (n) } & \multirow{2}{*}{$\mathrm{P}$} \\
\hline & $\leq 2.43$ & $>2.43$ & & \\
\hline \multicolumn{5}{|l|}{ Demographics } \\
\hline Number (n) & 300 & 256 & 556 & \\
\hline Age (y) & & & & 0.574 \\
\hline$\leq 60$ & $177(59.0)$ & $145(56.6)$ & $322(57.9)$ & \\
\hline$>60$ & $123(41.0)$ & $111(43.4)$ & $234(42.1)$ & \\
\hline Gender & & & & 0.002 \\
\hline Male & $211(70.3)$ & 209 (81.6) & $420(75.5)$ & \\
\hline Female & $89(29.7)$ & $47(18.4)$ & $136(24.5)$ & \\
\hline T stage & & & & 0.004 \\
\hline TO & $28(9.3)$ & 27 (10.5) & $55(9.9)$ & \\
\hline $\mathrm{T} 1$ & $48(16.0)$ & $21(8.2)$ & $69(12.4)$ & \\
\hline T2 & 57 (19.0) & $38(14.8)$ & $95(17.1)$ & \\
\hline T3 & $160(53.3)$ & $153(59.8)$ & $313(56.3)$ & \\
\hline $\mathrm{T} 4$ & $7(2.3)$ & $17(6.6)$ & $24(4.3)$ & \\
\hline $\mathrm{N}$ stage & & & & 0.355 \\
\hline No & $164(54.7)$ & $127(49.6)$ & $291(52.3)$ & \\
\hline N1 & $90(30.0)$ & $94(36.7)$ & $184(33.1)$ & \\
\hline N2 & 37 (12.3) & $26(10.2)$ & $63(11.3)$ & \\
\hline N3 & $9(3.0)$ & $9(3.5)$ & $18(3.2)$ & \\
\hline TNM stage & & & & 0.179 \\
\hline 0 & $28(9.3)$ & $26(10.2)$ & $54(9.7)$ & \\
\hline I & $46(15.3)$ & $22(8.6)$ & $68(12.2)$ & \\
\hline II & $104(34.7)$ & $90(35.2)$ & $194(34.9)$ & \\
\hline III & $115(38.3)$ & $110(43.0)$ & $225(40.4)$ & \\
\hline IV & $7(2.3)$ & $8(3.1)$ & $15(2.7)$ & \\
\hline Surgical approach & & & & 0.111 \\
\hline Sweet & $180(60.0)$ & $133(52.0)$ & $313(56.3)$ & \\
\hline Ivor-Lewis & $16(5.3)$ & $12(4.7)$ & $28(5.0)$ & \\
\hline McKeown & $104(34.7)$ & $111(43.4)$ & $215(38.7)$ & \\
\hline Complication & & & & 0.247 \\
\hline Yes & $42(14.0)$ & 45 (17.8) & 87 (15.6) & \\
\hline No & $258(86.0)$ & 211 (82.4) & $469(84.4)$ & \\
\hline
\end{tabular}

Table 1 (continued)
Table 1 (continued)

\begin{tabular}{|c|c|c|c|c|}
\hline \multirow{2}{*}{ Variables } & \multicolumn{2}{|c|}{ The level of NLR } & \multirow{2}{*}{ Total (n) } & \multirow{2}{*}{$\mathrm{P}$} \\
\hline & $\leq 2.43$ & $>2.43$ & & \\
\hline \multicolumn{4}{|c|}{ Blood pressure (mmHg) } & 0.134 \\
\hline$\leq 140 / 90$ & $262(87.3)$ & $212(82.8)$ & $474(85.3)$ & \\
\hline$>140 / 90$ & $38(12.7)$ & $44(17.2)$ & $82(14.7)$ & \\
\hline \multicolumn{4}{|l|}{ Pulse rate (/min) } & 0.236 \\
\hline$<60$ & $12(4.0)$ & $8(3.1)$ & $20(3.6)$ & \\
\hline $60-100$ & $283(94.3)$ & $238(93)$ & $521(93.7)$ & \\
\hline$>100$ & $5(1.7)$ & $10(3.9)$ & $15(2.7)$ & \\
\hline \multicolumn{4}{|c|}{ Respiratory rate (/min) } & 0.877 \\
\hline$<16$ & $67(22.3)$ & $55(21.5)$ & $122(21.9)$ & \\
\hline $16-20$ & $231(77.0)$ & $200(78.1)$ & $431(77.5)$ & \\
\hline$>20$ & $2(0.7)$ & $1(0.4)$ & $3(0.5)$ & \\
\hline \multicolumn{4}{|l|}{ Blood loss (mL) } & 0.093 \\
\hline$\leq 300$ & $284(94.7)$ & $233(91.0)$ & $517(93.0)$ & \\
\hline$>300$ & $16(5.3)$ & $23(9.0)$ & $39(7)$ & \\
\hline \multicolumn{4}{|l|}{$\begin{array}{l}\text { Hospitalization } \\
\text { days }\end{array}$} & 0.049 \\
\hline$\leq 24$ & $221(73.7)$ & $169(66.0)$ & $390(70.1)$ & \\
\hline$>24$ & 79 (26.3) & $87(34.0)$ & $166(29.9)$ & \\
\hline \multicolumn{5}{|l|}{$\begin{array}{l}\text { Postoperative } \\
\text { survival rate }\end{array}$} \\
\hline$\leq 6$ months & $18(6.0)$ & $25(9.8)$ & $43(7.7)$ & 0.098 \\
\hline$>6$ months & $282(94.0)$ & $231(90.2)$ & $513(92.3)$ & \\
\hline$\leq 3$ years & $145(48.3)$ & $142(55.5)$ & $287(51.6)$ & 0.093 \\
\hline$>3$ years & $155(51.7)$ & $114(44.5)$ & $269(48.4)$ & \\
\hline$\leq 5$ years & $252(84.0)$ & $223(87.1)$ & $475(85.4)$ & 0.3 \\
\hline$>5$ years & $48(16.0)$ & 33 (12.9) & 81 (14.6) & \\
\hline
\end{tabular}

NLR, neutrophil-to-lymphocyte ratio.

\section{Survival analysis of NLR}

We performed Kaplan-Meier analysis and log rank test to determine survival differences between the two groups classified by the NLR cutoff. The Kaplan-Meier survival curves show that patients with serum levels of NLR $\leq 2.43$ had better OS and DFS than patients with a serum NLR 
A

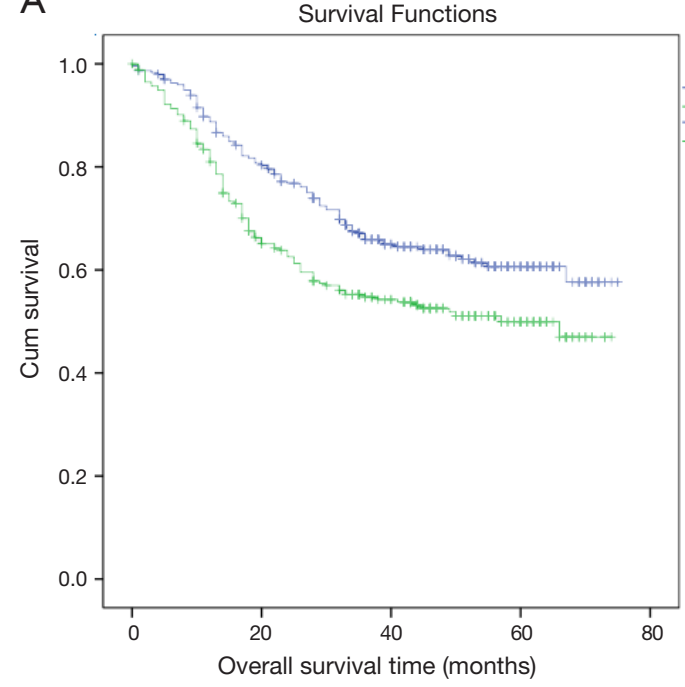

B

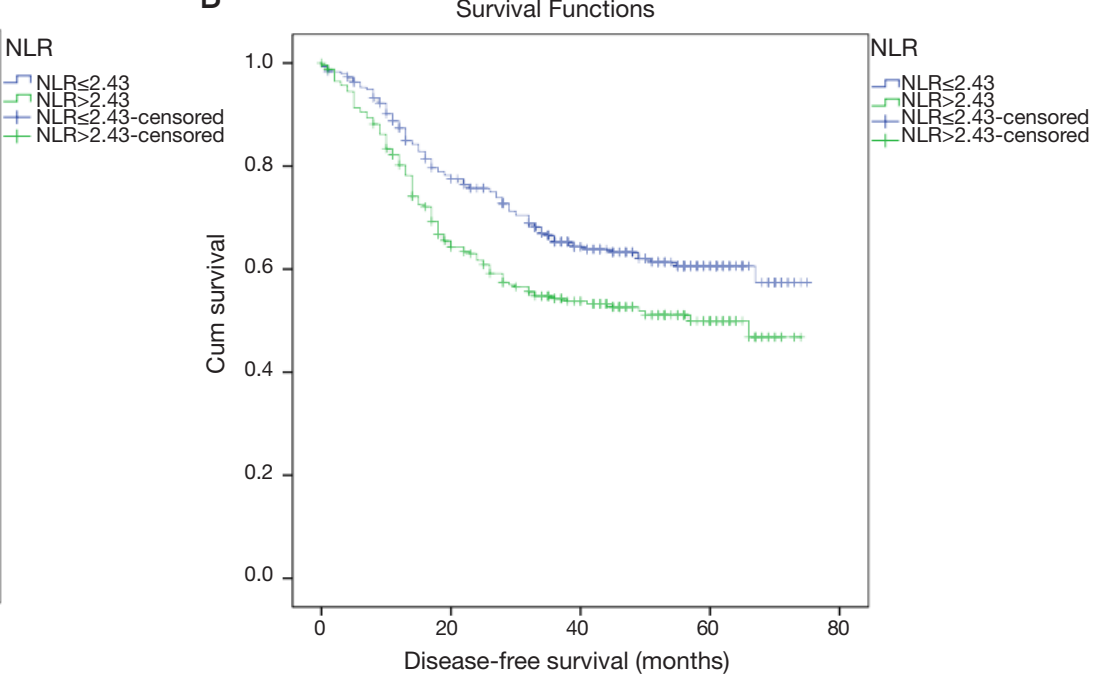

Figure 2 The Kaplan-Meier analysis of the survival differences classified by the cutoff of NLR. (A) Overall survival in the cohort grouped by the cutoff of NLR ( $\log$ rank =9.273, P=0.002); (B) disease-free survival in the cohort grouped by the cutoff of NLR (log rank =8.369, $\mathrm{P}=0.04)$. NLR, neutrophil-to-lymphocyte ratio.

Table 2 Comparison of the prognosis and recurrence for patients of NLR $\leq 2.43$ and $>2.43 \mathrm{ng} / \mathrm{mL}$

\begin{tabular}{|c|c|c|c|c|c|}
\hline Variables & $\mathrm{NLR} \leq 2.43 \mathrm{ng} / \mathrm{mL}$ & $\mathrm{NLR}>2.43 \mathrm{ng} / \mathrm{mL}$ & Total (n) & $\chi^{2}$ & $P$ \\
\hline Prognosis & & & & 7.183 & 0.009 \\
\hline Survival & 193 & 136 & 329 & & \\
\hline Dead & 107 & 120 & 227 & & \\
\hline Total (n) & 300 & 256 & 556 & & \\
\hline Mortality rate & $35.67 \%$ & $46.88 \%$ & & & \\
\hline Recurrence & & & & 1.191 & 0.275 \\
\hline No & 278 & 243 & 521 & & \\
\hline Yes & 22 & 13 & 35 & & \\
\hline Total (n) & 300 & 256 & 556 & & \\
\hline Recurrence rate & $7.33 \%$ & $5.08 \%$ & & & \\
\hline
\end{tabular}

NLR, neutrophil-to-lymphocyte ratio.

$>2.43 \mathrm{ng} / \mathrm{mL}(\log \operatorname{rank}=9.273, \mathrm{P}=0.002 ; \log \operatorname{rank}=8.369$, $\mathrm{P}=0.004$; Figure $2 A$ and $B$, respectively). The 5 -year OS rates were $16 \%$ and $12.9 \%$, and the median survival times were 54.14 and 45.71 months for those with a NLR $\leq 2.43 \mathrm{ng} / \mathrm{mL}$ and NLR $>2.43 \mathrm{ng} / \mathrm{mL}$ respectively ( $\mathrm{P}=0.002$, Figure 2$)$. Patients with a NLR $\leq 2.43 \mathrm{ng} / \mathrm{mL}$ had a higher OS than those with a NLR $>2.43 \mathrm{ng} / \mathrm{mL}(\mathrm{P}=0.036)$ (Table 2, Figure 3).
Furthermore, the analysis of survival time and preoperative NLR level is necessary to perform.

According to the NLR cutoff, we divided the patients into two groups. It is showed that the preoperative NLR, T stage, $\mathrm{N}$ stage, surgical approach and gender are associated with OS and DFS in the univariate Cox regression analysis (all $\mathrm{P}<0.05)$ (Table S1). 


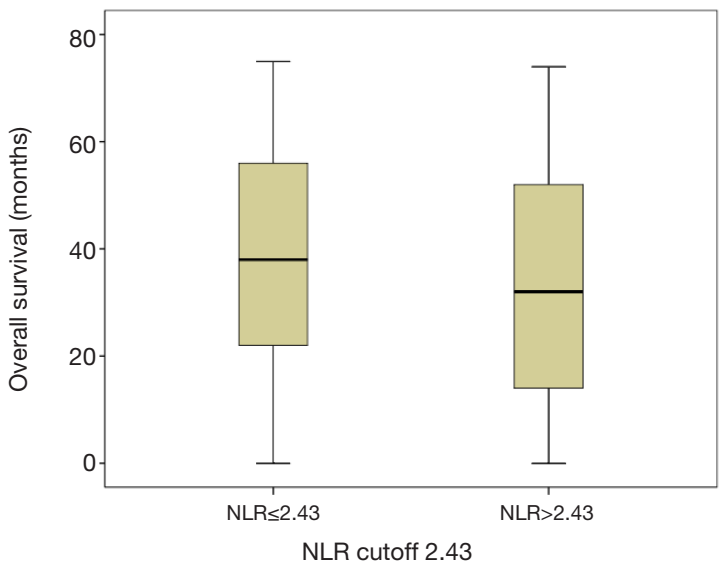

Figure 3 Preoperative serum levels of NLR and ESCC patient overall survival.

\section{Multivariate analysis of independent prognostic indicators patient characteristics and $O S$}

We performed Cox multivariate analysis to determine the independent predictive indexes. As shown in Table 3, multivariate Cox regression analysis showed that preoperative NLR $>2.43 \mathrm{ng} / \mathrm{mL}$ had shorter OS compared to patients with a serum NLR $\leq 2.43$ (HR $=1.365 ; 95 \%$ CI: $1.032-1.805 ; \mathrm{P}=0.029)$. In addition, the following variables were independent factors associated with low survival: T4 stage, $\mathrm{N}$ metastasis, and age $>60$ (Table 3).

\section{Prognostic significance of NLR in different TNM stages of $E C$}

The relationship between TNM and NLR in predicting the prognosis of EC after surgery is presented in Table 4. As shown in Figure 4, multivariate analysis showed that the preoperative NLR was significantly correlated with the OS in stages 0 , I and II middle thoracic ESCC(stage 0: $\mathrm{P}=0.012$ for OS, stage I: $\mathrm{P}=0.046$ for OS, stage II: $\mathrm{P}=0.044$ for OS; Figure $4 A, B, C)$. However, multivariate analysis showed that there was no significant relationship between the preoperative NLR and the OS in patients with stage III and IV middle thoracic ESCC $(\mathrm{P}=0.583$ for OS and $\mathrm{P}=0.208$ for OS, Figures 4D,E). A total of 555, and 230 cases died in TNM stages 0, I, II, III and IV, of which the mortality rate in those with a NLR $\leq 2.43 \mathrm{ng} / \mathrm{mL}$ was $21.4 \%(6 / 28), 17.4 \%$ (8/46), 24\% (25/104), 54.8\% (63/115) and $85.7 \%(6 / 7)$ respectively. The mortality rate in those with NLR $>2.43$ $\mathrm{ng} / \mathrm{mL}$ was $53.8 \%$ (14/26), 40.9\% (9/22), 36.0\% (32/89), $56.4 \%(62 / 110)$ and $62.5(5 / 8)$, respectively (Table 4).

\section{Discussion}

Our study concluded that the optimum cutoff point for preoperative NLR was $2.43 \mathrm{ng} / \mathrm{mL}$ for OS and DFS as verified by ROC curves in middle thoracic ESCC, and the area under the curve was 0.553 for the NLR in predicting prognosis in patients with middle thoracic ESCC (Figure 1). Therefore, we defined the cutoff values as $2.43 \mathrm{ng} / \mathrm{mL}$ as inferred by ROC analysis, which was lower than that in previous studies (25-27).

Our results showed that increased NLR is associated with lower OS and shorter DFS in patients with mid thoracic ESCC. In addition, Cox regression indicated that a NLR $>2.43 \mathrm{ng} / \mathrm{mL}$ was an independent predictor of poor OS (Table 3).

Univariate analysis also showed that the factors that were significantly related to the OS and DFS were preoperative NLR, T stage, $\mathrm{N}$ stage, surgical approach, and gender (all $\mathrm{P}<0.05$ ) (Table S1). NLR >2.43 ng/mL, higher T stage, higher $\mathrm{N}$ stage, Mecknown approach, and male were risk factors reduced OS and DFS in middle thoracic ESCC by univariate analysis (Table S1). However, anesthetic factors including blood pressure, pulse rate, respiratory rate, blood loss and other factors like hospitalization days, complications and age were not significantly associated with OS and DFS (all P>0.05) (Table S1).

Multivariate Cox Regression Analysis also shows that prognostic factors including preoperative NLR, T stage, N stage, surgical approach, gender and age also influence the OS and DFS in middle thoracic ESCC. NLR $>2.43 \mathrm{ng} / \mathrm{mL}$, higher $\mathrm{T}$ stage, higher $\mathrm{N}$ stage, Mecknown approach, male and age $>60$ reduced OS and DFS in middle thoracic ESCC (all $\mathrm{P}<0.05$ ) (Table 3). However, there is no significant influence of anesthetic factors including blood pressure, pulse rate, respiratory rate, blood loss and other factors like hospitalization days and complications on OS and DFS by Multivariate Cox Regression Analysis (all P>0.05) (Table 3).

We also measured the relationship between TNM stage and NLR in the prognosis prediction of TNM stage $0,1,2$ and 3 , and we found that patients with NLR $>2.43 \mathrm{ng} / \mathrm{mL}$ had a worse prognosis than those with NLR $\leq 2.43 \mathrm{ng} / \mathrm{mL}$. The mortality rates in patients (NLR $>2.43 \mathrm{ng} / \mathrm{mL}$ ) with stages $0,1,2$ and 3 were $53.8 \%, 40.9 \%, 36 \%$ and $56.4 \%$, respectively (Table 4).

Many previous studies have used multicenter databases to demonstrate that haematological inflammatory markers can help predict survival in patients with different types of cancer $(11,24,28,29)$. In addition, several previous 
Table 3 Multivariate Cox regression analysis of prognostic factors influencing overall survival and disease-free survival

\begin{tabular}{|c|c|c|c|c|c|c|}
\hline Variables & \multicolumn{3}{|c|}{ Overall survival } & \multicolumn{3}{|c|}{ Disease-free survival } \\
\hline Cutoff of NLR (ng/mL) & & & 0.029 & & & 0.045 \\
\hline$N L R \leq 2.43$ & 1 & & & 1 & & \\
\hline $\mathrm{NLR}>2.43$ & 1.365 & $1.032-1.805$ & 0.029 & 1.331 & $1.007-1.759$ & 0.045 \\
\hline TO & 1 & & & 1 & & \\
\hline $\mathrm{T} 1$ & 0.475 & $0.238-0.946$ & 0.034 & 0.469 & $0.236-0.932$ & 0.031 \\
\hline T2 & 0.583 & $0.322-1.055$ & 0.075 & 0.571 & $0.315-1.036$ & 0.065 \\
\hline T3 & 0.958 & $0.574-1.598$ & 0.869 & 0.957 & $0.573-1.599$ & 0.867 \\
\hline No & 1 & & & 1 & & \\
\hline $\mathrm{N} 1$ & 1.869 & $1.360-2.569$ & $<0.001$ & 1.902 & $1.384-2.615$ & $<0.001$ \\
\hline N2 & 2.629 & $1.740-3.972$ & $<0.001$ & 2.550 & $1.684-3.860$ & $<0.001$ \\
\hline N3 & 5.054 & $3.20-9.79$ & $<0.001$ & 4.741 & $2.711-8.293$ & $<0.001$ \\
\hline Surgical approach & & & 0.002 & & & 0.003 \\
\hline Sweet & 0.237 & $0.108-0.520$ & $<0.001$ & 0.249 & $0.113-0.544$ & 0.001 \\
\hline Ivor-Lewis & 0.211 & $0.082-0.544$ & 0.001 & 0.225 & $0.087-0.580$ & 0.002 \\
\hline Mecknown & 0.213 & $0.097-0.468$ & $<0.001$ & 0.224 & $0.102-0.492$ & $<0.001$ \\
\hline$>60$ & 1.328 & $1.006-1.753$ & 0.045 & 1.391 & $1.064-1.818$ & 0.016 \\
\hline Gender & & & 0.044 & & & 0.033 \\
\hline Female & 1 & & & 1 & & \\
\hline Male & 1.453 & $1.011-2.090$ & 0.044 & 1.484 & $1.032-2.133$ & 0.033 \\
\hline Blood pressure $(\mathrm{mmHg})$ & & & 0.781 & & & 0.999 \\
\hline \multicolumn{7}{|l|}{$\leq 140 / 90$} \\
\hline \multicolumn{7}{|l|}{$>140 / 90$} \\
\hline Pulse rate (/min) & & & 0.589 & & & 0.525 \\
\hline \multicolumn{7}{|l|}{$<60$} \\
\hline \multicolumn{7}{|l|}{$60-100$} \\
\hline$>100$ & & & & & & \\
\hline
\end{tabular}

Table 3 (continued) 
Table 3 (continued)

\begin{tabular}{|c|c|c|c|c|c|c|}
\hline Variables & \multicolumn{3}{|c|}{ Overall survival } & \multicolumn{3}{|c|}{ Disease-free survival } \\
\hline Respiratory rate (/min) & & & 0.596 & & & 0.621 \\
\hline \multicolumn{7}{|l|}{$<16$} \\
\hline \multicolumn{7}{|l|}{$16-20$} \\
\hline Blood loss (mL) & & & 0.582 & & & 0.592 \\
\hline \multicolumn{7}{|l|}{$\leq 300$} \\
\hline \multicolumn{7}{|l|}{$>300$} \\
\hline Hospitalization days & & & 0.796 & & & 0.842 \\
\hline
\end{tabular}

$\mathrm{HR}$, hazard ratio; Cl, confidence interval; NLR, neutrophil-to-lymphocyte ratio.

Table 4 Relationship between TNM and NLR in predicting prognosis

\begin{tabular}{|c|c|c|c|c|c|c|}
\hline TNM stage & NLR & \multicolumn{2}{|c|}{ Prognosis, n (\%) } & Total (n) & $\chi^{2}$ & $P$ \\
\hline \multirow[t]{2}{*}{0} & $\leq 2.43 \mathrm{ng} / \mathrm{mL}$ & $22(78.6)$ & $6(21.4)$ & 28 & 6.256 & 0.012 \\
\hline & $>2.43 \mathrm{ng} / \mathrm{mL}$ & $12(46.2)$ & $14(53.8)$ & 26 & & \\
\hline 1 & $\leq 2.43 \mathrm{ng} / \mathrm{mL}$ & $38(82.6)$ & $8(17.4)$ & 46 & 3.965 & 0.046 \\
\hline \multirow[t]{2}{*}{2} & $\leq 2.43 \mathrm{ng} / \mathrm{mL}$ & $79(76.0)$ & $25(24.0)$ & 104 & 4.048 & 0.044 \\
\hline & $>2.43 \mathrm{ng} / \mathrm{mL}$ & $57(64.0)$ & $32(36.0)$ & 89 & & \\
\hline \multirow[t]{2}{*}{3} & $\leq 2.43 \mathrm{ng} / \mathrm{mL}$ & $52(45.2)$ & $63(54.8)$ & 115 & 0.302 & 0.583 \\
\hline & $>2.43 \mathrm{ng} / \mathrm{mL}$ & $48(43.6)$ & $62(56.4)$ & 110 & & \\
\hline
\end{tabular}

NLR, neutrophil-to-lymphocyte ratio.

studies showed that an elevated preoperative NLR was a poor prognostic marker for ECSS patients after surgical resection. Yodying et al. reported that there was significant correlation between NLR level and clinical characteristics of patients (30). Xue et al. revealed that NLR was related to the vascular invasion of liver cancer (31). Plus, Yodying et al. claimed that an elevated NLR was associated with tumor invasion and LN metastasis (30). However, we demonstrated that elevated preoperative NLR predicts poor prognostic in middle thoracic ESCC by univariate and multivariate Cox regression analysis, which has never been discussed in previous studies.

We believe that retrospective studies using a single institute's database may produce more homogeneous results than multicenter databases, considering that the surgical procedures of a single institute are more likely to be consistent.

A previous research reported that NLR levels is useful 

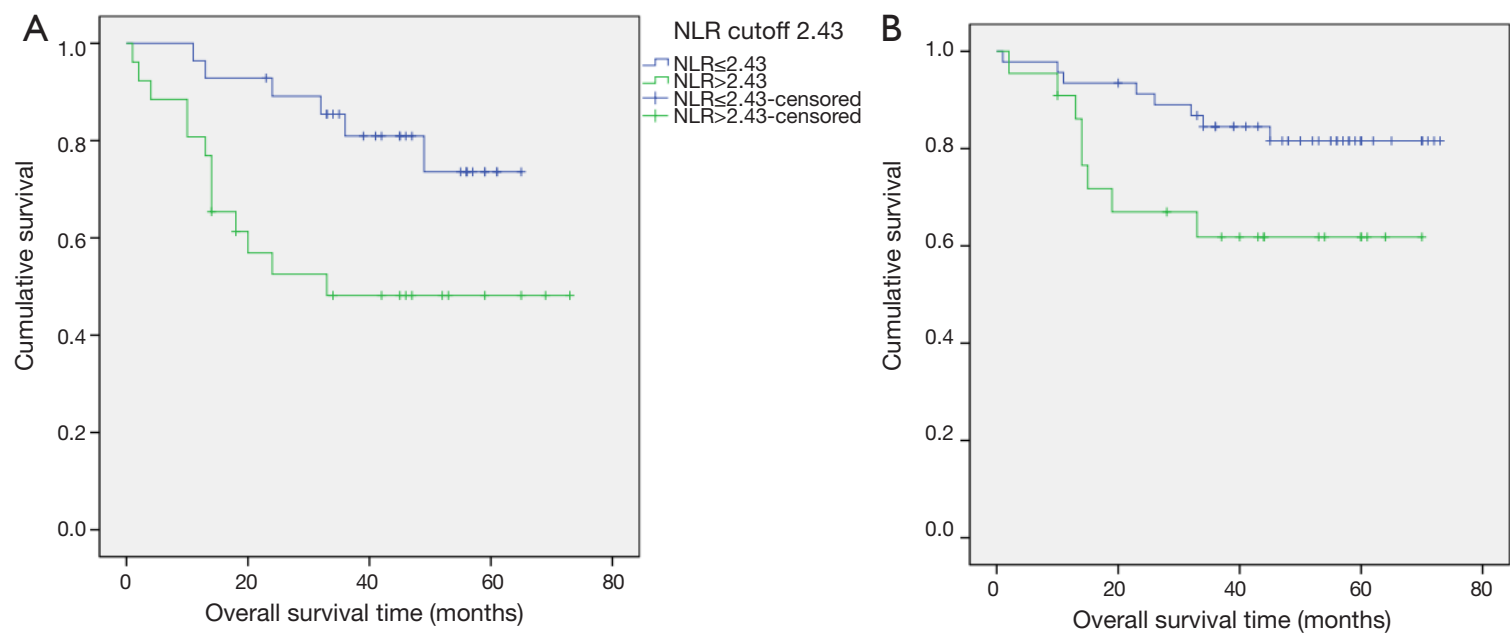

NLR cutoff 2.43

C

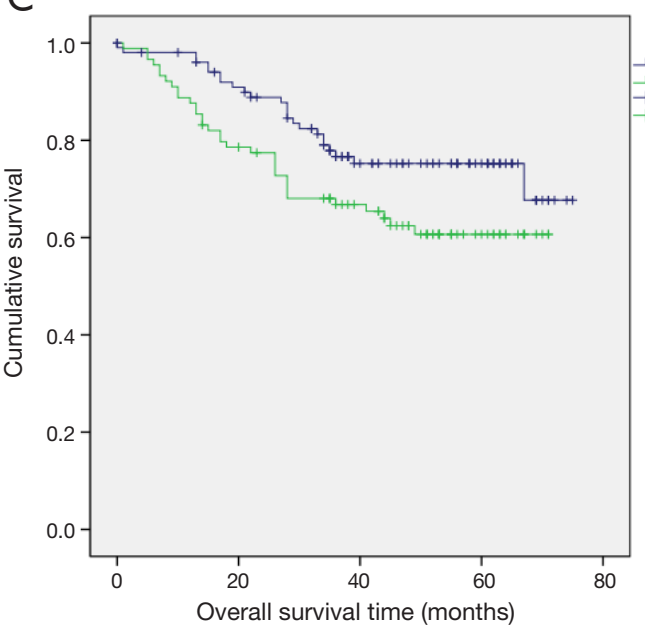

NLR cutoff 2.43
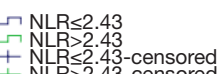
$+\mathrm{NLR} \leq 2.43$-censored

D

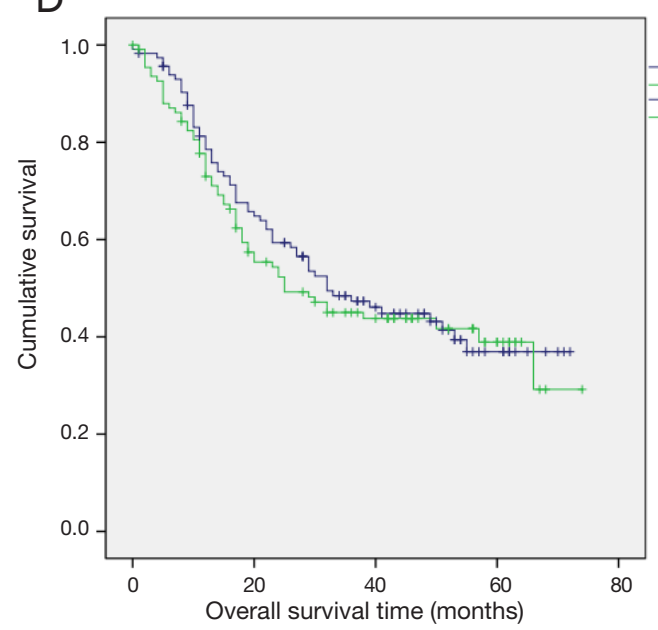

NLR cutoff 2.43 $\rightarrow N L R \leq 2.43$ $-\mathrm{NLR}<2.43$-censored

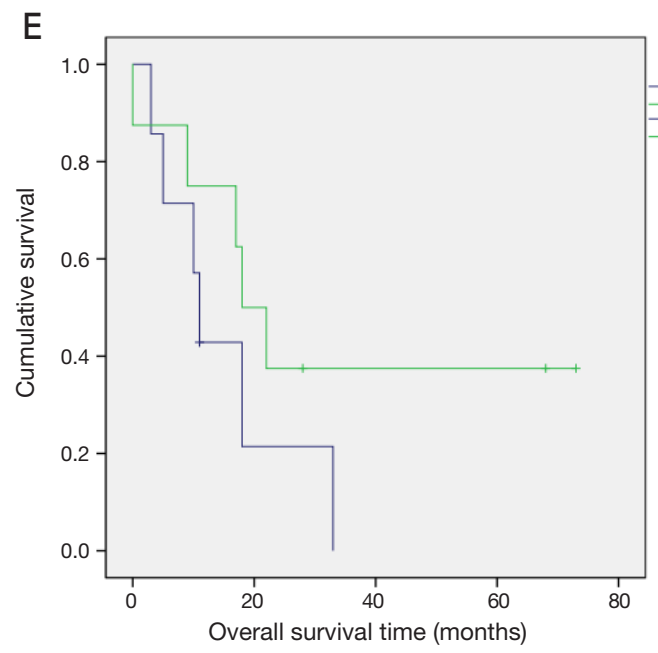

NLR cutoff 2.43

$\neg N L R \leq 2.43$

$+N L R \leq 2.43$-censored

Figure 4 Survival curves of patients with esophageal cancer in NLR. (A) OS curve of patients with stage 0 NSCLC with esophageal cancer (log-rank test, $\mathrm{P}=0.012$ ); (B) OS curve of patients with stage I esophageal cancer (log-rank test, $\mathrm{P}=0.046$ ); (C) OS curve of patients with stage II esophageal cancer (log-rank test, $\mathrm{P}=0.044$ ); (D) OS curve of patients with stage III esophageal cancer (log-rank test, $\mathrm{P}=0.583$ ); (E) OS curve of patients with stage IV esophageal cancer (log-rank test, $\mathrm{P}=0.208$ ). NLR, neutrophil-to-lymphocyte ratio; NSCLC, non-small cell lung cancer; OS, overall survival. 
for detecting relapse and evaluating responses to treatment in postoperative patients with gastric and ECs (16). Previous studies have reported that elevated NLR levels was an independent predictive factors for poor OS in EC patients, while the optimal cut off and preoperative NLR value in middle thoracic ESCC was uncertain $(30,32)$. We did not define the cutoff as the normal upper limit, as most of the patients included in our study were in the early and medial stages of the disease and were primarily eligible for surgical treatment, which justifies the lower cutoff values.

There are several limitations should be clarified in this study. First of all, our study is a single center retrospective analysis, so the results of this study may be biased. Secondly, this study retrospectively analyzed 562 esophageal carcinoma patients who underwent radical esophagectomy from January 2010 to December 2012 at the Sun Yatsen University Cancer Center. The predictive value of preoperative NLR for middle thoracic ESCC needs further study with larger samples. Thirdly, some of our findings are inconsistent with the literature. Our results should be verified by additional studies.

The strength of the current study was that we studied ESCC patients from a single institution using relatively standard surgical techniques to ensure accurate pathological staging and the radical resection. The representative sample and the considerably high follow-up rate guaranteed the reliability of our results. In addition, our results showed a significant positive correlation between NLR and clinical features of ESCC, such as gender and T stage. Therefore, NLR may have a wider clinical application in the prognosis evaluation of ESCC, and it may be helpful to stratify patients and determine individualized treatment plans in the future.

In conclusion, we show that high pre-treatment NLR is associated with poor prognosis in ESCC patients, while the survival rate in high serum NLR patients may be relatively low. NLR can be used as a promising prognostic indicator, because it can be measured in routine blood routine test in clinical practice, and it is convenient, economical, and reproducible. Future prospective studies with larger populations are needed to validate our findings and to determine the potential mechanisms underlying the prognostic value of the NLR.

\section{Acknowledgments}

We would also like to thank all investigators helped in data collection and analysis. We are also grateful to all who reviewed and commented on an early draft of the paper.

Funding: This paper was supported by the National Natural Science Foundation (81871986).

\section{Footnote}

Conflicts of Interest: The authors have no conflicts of interest to declare.

Ethical Statement: The authors are accountable for all aspects of the work in ensuring that questions related to the accuracy or integrity of any part of the work are appropriately investigated and resolved. Written informed consent was obtained from all patients. This study was approved by the Ethics Committee of Sun Yat-sen University Cancer Center (approval number: GZR 2018-120).

Open Access Statement: This is an Open Access article distributed in accordance with the Creative Commons Attribution-NonCommercial-NoDerivs 4.0 International License (CC BY-NC-ND 4.0), which permits the noncommercial replication and distribution of the article with the strict proviso that no changes or edits are made and the original work is properly cited (including links to both the formal publication through the relevant DOI and the license). See: https://creativecommons.org/licenses/by-nc-nd/4.0/.

\section{References}

1. Chen $\mathrm{W}$, Zheng R, Baade PD, et al. Cancer statistics in China, 2015. CA Cancer J Clin 2016;66:115-32.

2. Bray F, Ferlay J, Soerjomataram I, et al. Global cancer statistics 2018: GLOBOCAN estimates of incidence and mortality worldwide for 36 cancers in 185 countries. CA Cancer J Clin 2018;68:394-424.

3. Rashid F, Waraich N, Bhatti I, et al. A pre-operative elevated neutrophil: lymphocyte ratio does not predict survival from oesophageal cancer resection. World J Surg Oncol 2010;8:1.

4. Wang CY, Hsieh MJ, Chiu YC, et al. Higher serum C-reactive protein concentration and hypoalbuminemia are poor prognostic indicators in patients with esophageal cancer undergoing radiotherapy. Radiother Oncol 2009;92:270-5.

5. Idzko M, Ferrari D, Eltzschig HK. Nucleotide signalling during inflammation. Nature 2014;509:310-7. 
6. Proctor MJ, Morrison DS, Talwar D, et al. A comparison of inflammation-based prognostic scores in patients with cancer. A Glasgow Inflammation Outcome Study. Eur J Cancer 2011;47:2633-41.

7. Proctor MJ, Talwar D, Balmar SM, et al. The relationship between the presence and site of cancer, an inflammationbased prognostic score and biochemical parameters. Initial results of the Glasgow Inflammation Outcome Study. Br J Cancer 2010;103:870-6.

8. Gomez D, Morris-Stiff G, Toogood GJ, et al. Impact of systemic inflammation on outcome following resection for intrahepatic cholangiocarcinoma. J Surg Oncol 2008;97:513-8.

9. Moses K, Brandau S. Human neutrophils: Their role in cancer and relation to myeloid-derived suppressor cells. Semin Immunol 2016;28:187-96.

10. Cho H, Hur HW, Kim SW, et al. Pre-treatment neutrophil to lymphocyte ratio is elevated in epithelial ovarian cancer and predicts survival after treatment. Cancer Immunol Immunother 2009;58:15-23.

11. Thavaramara T, Phaloprakarn C, Tangjitgamol S, et al. Role of neutrophil to lymphocyte ratio as a prognostic indicator for epithelial ovarian cancer. J Med Assoc Thai 2011;94:871-7.

12. Kim HS, Choi HY, Lee M, et al. Systemic Inflammatory Response Markers and CA-125 Levels in Ovarian Clear Cell Carcinoma: A Two Center Cohort Study. Cancer Res Treat 2016;48:250-8.

13. Supoken A, Kleebkaow P, Chumworathayi B, et al. Elevated preoperative platelet to lymphocyte ratio associated with decreased survival of women with ovarian clear cell carcinoma. Asian Pac J Cancer Prev 2014;15:10831-6.

14. Grivennikov SI, Greten FR, Karin M. Immunity, inflammation, and cancer. Cell 2010;140:883-99.

15. Märkl B, Wieberneit J, Kretsinger H, et al. Number of Intratumoral T Lymphocytes Is Associated With Lymph Node Size, Lymph Node Harvest, and Outcome in Node-Negative Colon Cancer. Am J Clin Pathol 2016;145:826-36.

16. Noble F, Mellows T, McCormick ML, et al. Tumour infiltrating lymphocytes correlate with improved survival in patients with oesophageal adenocarcinoma. Cancer Immunol Immunother 2016;65:651-62.

17. Muroya T, Wajima N, Ogasawara H, et al. [The Impact of Neutrophil-Lymphocyte Ratio on the Prognosis in Patients Who Underwent Esophagectomy for Esophageal
Cancer]. Gan To Kagaku Ryoho 2017;44:912-914.

18. Templeton AJ, McNamara MG, Seruga B, et al. Prognostic role of neutrophil-to-lymphocyte ratio in solid tumors: a systematic review and meta-analysis. J Natl Cancer Inst 2014;106:dju124.

19. Paramanathan A, Saxena A, Morris DL. A systematic review and meta-analysis on the impact of pre-operative neutrophil lymphocyte ratio on long term outcomes after curative intent resection of solid tumours. Surg Oncol 2014;23:31-9.

20. Zhang X, Zhang W, Feng LJ. Prognostic significance of neutrophil lymphocyte ratio in patients with gastric cancer: a meta-analysis. PLoS One 2014;9:e111906.

21. Kosumi K, Baba Y, Ishimoto T, et al. Neutrophil/ lymphocyte ratio predicts the prognosis in esophageal squamous cell carcinoma patients. Surg Today 2016;46:405-13.

22. Sharaiha RZ, Halazun KJ, Mirza F, et al. Elevated preoperative neutrophil:lymphocyte ratio as a predictor of postoperative disease recurrence in esophageal cancer. Ann Surg Oncol 2011;18:3362-9.

23. Rice TW, Ishwaran H, Hofstetter WL, et al, AppersonHansen C, Blackstone EH. Recommendations for pathologic staging (pTNM) of cancer of the esophagus and esophagogastric junction for the 8th edition AJCC/ UICC staging manuals. Dis Esophagus 2016;29:897-905.

24. Agha RA, Borrelli MR, Vella-Baldacchino M, et al. The STROCSS statement: Strengthening the Reporting of Cohort Studies in Surgery. Int J Surg 2017;46:198-202.

25. Yuan D, Zhu K, Li K, et al. The preoperative neutrophil-lymphocyte ratio predicts recurrence and survival among patients undergoing R0 resections of adenocarcinomas of the esophagogastric junction. J Surg Oncol 2014;110:333-40.

26. Pirozzolo G, Gisbertz SS, Castoro C, et al. Neutrophilto-lymphocyte ratio as prognostic marker in esophageal cancer: a systematic review and meta-analysis. J Thorac Dis 2019;11:3136-3145.

27. Toyokawa T, Kubo N, Tamura T, et al. The pretreatment Controlling Nutritional Status (CONUT) score is an independent prognostic factor in patients with resectable thoracic esophageal squamous cell carcinoma: results from a retrospective study. BMC Cancer 2016;16:722.

28. Wang Z, Zhan P, Lv Y, et al. Prognostic role of pretreatment neutrophil-to-lymphocyte ratio in non-small cell lung cancer patients treated with systemic therapy: a meta-analysis. Transl Lung Cancer Res 2019;8:214-26. 
29. Han F, Liu Y, Cheng S, et al. Diagnosis and survival values of neutrophil-lymphocyte ratio (NLR) and red blood cell distribution width (RDW) in esophageal cancer. Clin Chim Acta 2019;488:150-8.

30. Yodying H, Matsuda A, Miyashita M, et al. Prognostic Significance of Neutrophil-to-Lymphocyte Ratio and Platelet-to-Lymphocyte Ratio in Oncologic Outcomes of Esophageal Cancer: A Systematic Review and Metaanalysis. Ann Surg Oncol 2016;23:646-54.

Cite this article as: Hu J, Chen D, Wu S, Chen Y, Li R, Miao H, Wen Z. Prognostic significance of neutrophil-to-lymphocyte ratio in middle thoracic esophageal squamous cell carcinoma patients undergoing radical esophagectomy. J Thorac Dis 2020;12(3):363-374. doi: 10.21037/jtd.2020.01.31
31. Xue TC, Jia QA, Ge NL, et al. Imbalance in systemic inflammation and immune response following transarterial chemoembolization potentially increases metastatic risk in huge hepatocellular carcinoma. Tumour Biol 2015;36:8797-803.

32. Gao GD, Sun B, Wang XB, et al. Neutrophil to lymphocyte ratio as prognostic indicator for patients with esophageal squamous cell cancer. Int J Biol Markers 2017;32:e409-e414. 
Supplementary

Table S1 Univariate Cox regression analysis of prognostic factors influencing overall survival and disease-free survival

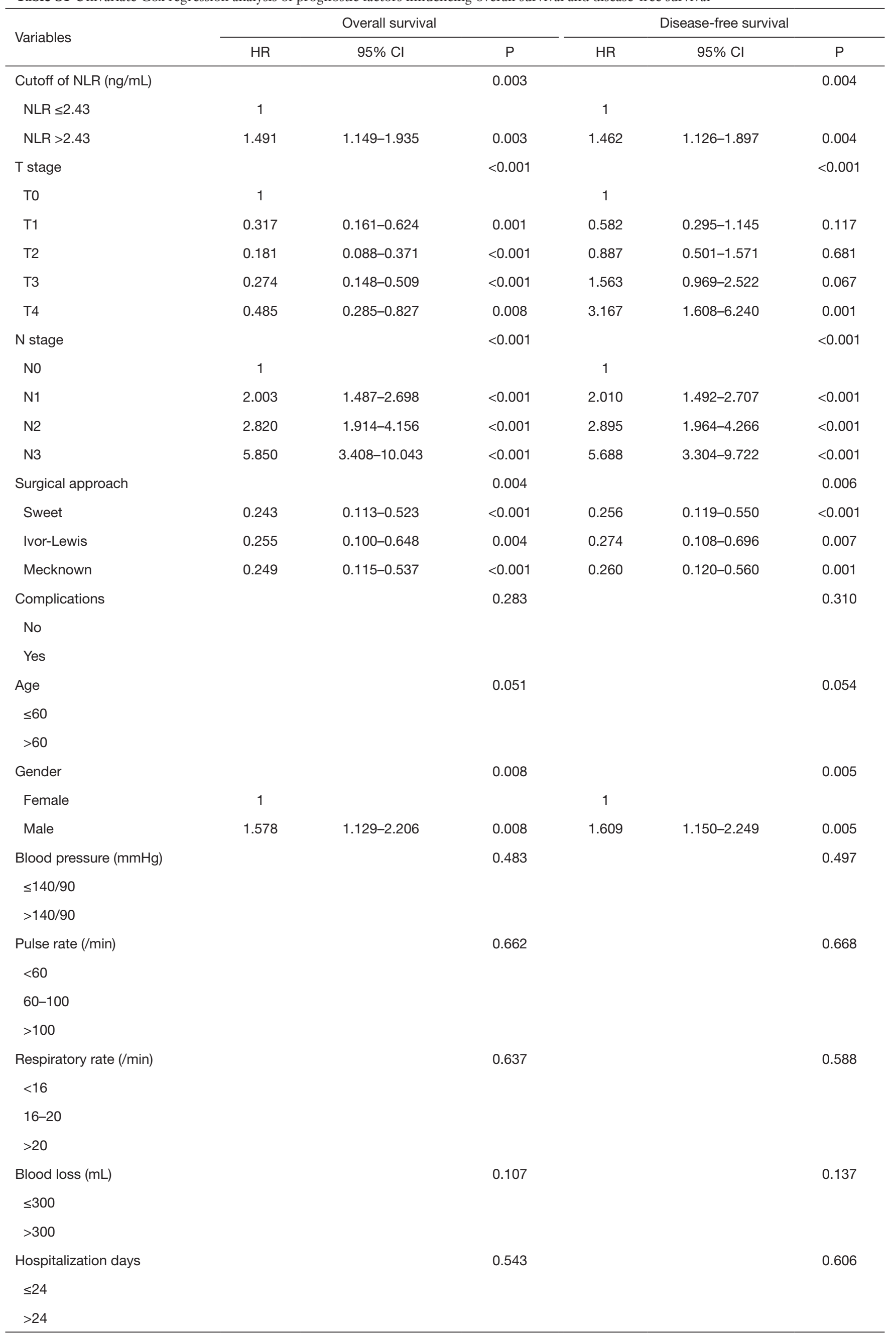

$\mathrm{HR}$, hazard ratio; $\mathrm{Cl}$, confidence interval; NLR, neutrophil-to-lymphocyte ratio. 